CARTA AL EDITOR

\section{ACTIVIDADES DE INMUNIZACIÓN EN EL CONTEXTO DE LA PANDEMIA POR LA COVID-19 EN LATINOAMÉRICA}

\section{IMMUNIZATION ACTIVITIES IN THE CONTEXT OF THE COVID-19 PANDEMIC IN LATIN AMERICA}

\author{
Noé Atamari-Anahui (1) 1,2,a, \\ Nadin Melina Conto-Palomino (1) 3,4,a, \\ César Johan Pereira-Victorio (1) 5,6

\footnotetext{
1 Universidad San Ignacio de Loyola, Vicerrectorado de Investigación, Unidad de Investigación para la Generación y Síntesis de Evidencias en Salud, Lima, Perú. (AMERINSN), Instituto Nacional de Salud del Niño-Breña, Lima, Perú.

3 Servicio de Pediatría, Hospital Nacional Arzobispo Loayza, Lima, Perú. Nacional Mayor de San Marcos, Lima, Perú.

5 Universidad Continental, Lima, Perú.

a Médico cirujano; ${ }^{\text {b }}$ médico cirujano, magister en Salud Pública.
} \\ 2 Asociación de Médicos Residentes del Instituto Nacional de Salud del Niño \\ 4 Escuela de Posgrado, Facultad de Medicina Humana, Universidad
}

Sr. Editor: Del 25 de abril al 2 de mayo del 2020 se programó la XVIII Campaña de la Semana de Vacunación en las Américas. Sin embargo, en el contexto de la actual pandemia, es posible que varios países de Latinoamérica hayan retrasado esta actividad ${ }^{(1)}$, cuyo objetivo es mejorar las coberturas de vacunación en la población en general, con especial atención en quienes se encuentran en situación de vulnerabilidad, tienen un acceso limitado a los servicios de salud y pertenecen a grupos de alto riesgo ${ }^{(2)}$.

La pandemia por la enfermedad por coronavirus (COVID-19) está afectando los sistemas de salud en los países de la región. Asimismo, afecta las coberturas de vacunación en varios países del mundo ${ }^{(3)}$. Esto se debe a la priorización de actividades sanitarias, sociales y económicas que han tomado los países para contener, controlar y mitigar la pandemia. Por ejemplo, en el Perú las medidas de distanciamiento social y la suspensión temporal del Programa Nacional de Inmunizaciones (PNI) han propiciado dificultades de movilización de personas y la desconfianza del personal sanitario y de la población sobre el uso de las vacunas. Esto ha generado la reacción inmediata del PNI mediante actividades de promoción para contener un probable perjuicio en las coberturas de vacunación.

Citar como: Atamari-Anahui N, Conto-Palomino NM, Pereira-Victorio CJ. Actividades de inmunización en el contexto de la pandemia por la COVID-19 en Latinoamérica. Rev Peru Med Exp Salud Publica. 2020;37(4):773-5. doi https://doi.org/10.17843/rpmesp.2020.374.5758.

Correspondencia: Noé Atamari-Anahui; Avenida La Fontana 550 La Molina Lima, Perú; noe.atamari@gmail.com

Recibido: 08/05/2020 Aprobado: 23/07/2020 En línea: 31/08/2020
En el contexto de la declaración de pandemia, se realizó el presente estudio con el objetivo de describir las actividades de promoción, desarrollo de estrategias de vacunación y preferencia vacunal en el mes previo y durante la Semana de Vacunación en las Américas (SVA) 2020. Cada año en la región, entre los meses de abril y mayo, se propicia la campaña de la SVA con la intención de mejorar las coberturas de vacunación y favorecer la confianza de la población en las vacunas como estrategia para salvar vidas ${ }^{(2)}$.

Para desarrollar el estudio, entre el 11 de marzo y el 7 de mayo del 2020 se realizó una búsqueda no sistemática a través de la consulta de las páginas web oficiales de los ministerios de Salud de los 20 países latinoamericanos. Se consideraron documentos elegibles a los incluidos en las resoluciones ministeriales, boletines y diarios oficiales, planes de contingencia y comunicados oficiales, notas de prensa, boletines informativos, informes técnicos o directivas nacionales, cuyo objetivo fuera desarrollar actividades de inmunización a partir de la fecha de declaración de pandemia por la COVID-19 (11 de marzo). Se consideró, además, el país de origen, la fecha de publicación de la actividad, el haber realizado alguna estrategia de vacunación y la preferencia vacunal.

De los 20 países latinoamericanos evaluados, nueve (45\%) publicaron algún comunicado sobre el desarrollo o la aplicación de inmunizaciones después de la declaración de la pandemia (Tabla 1). La mayoría de los países, aparte de promover acciones para la inmunización en los niños y grupos de riesgo, hicieron énfasis en la vacunación contra la influenza. Brasil recomendó también la vacunación contra el sarampión y la fiebre amarilla, y el Perú y Colombia, contra el neumococo. Todos los países contaron con estrategias de vacunación según su propio contexto epidemiológico.

Actualmente, las coberturas de vacunación en países latinoamericanos están por debajo de las recomendaciones de la Organización Panamericana de la Salud (OPS) y la OMS ${ }^{(4)}$. Es posible que la pandemia por la COVID-19 perjudique aún más las coberturas en todos los países afectados. Algunas organizaciones de salud han mostrado su preocupación por la posible reemergencia de enfermedades prevenibles con vacunas y han sugerido reiniciar las inmunizaciones en las poblaciones vulnerables según el contexto de cada país ${ }^{(3)}$. Por un lado, se ha observado que la mayoría de los países han priorizado la vacunación contra la influenza, probablemente relacionado a la temporada de bajas temperaturas en el hemisferio sur, periodo en el que se incrementan la incidencia y la mortalidad por enfermedades respiratorias con mayor afectación de la población vulnerable, menores de 5 años y mayores de 65 años. Por lado, al 17 de abril del 2020 en Latinoamérica, se han reportado 1104 casos confirmados de sarampión, siendo Brasil y México los más afectados ${ }^{(5)}$. Esto refleja las inadecuadas coberturas de vacunación que tienen los países de la región y que ponen en riesgo la aparición de brotes de enfermedades prevenibles con vacunas. 
Tabla 1. Actividades de inmunización en el contexto de la pandemia por la COVID-19 publicadas en las páginas web oficiales de los ministerios de Salud de nueve países de Latinoamérica.

\begin{tabular}{|c|c|c|c|c|}
\hline País & $\begin{array}{c}\text { Fecha de } \\
\text { publicación }\end{array}$ & $\begin{array}{l}\text { Estrategia de } \\
\text { vacunación }\end{array}$ & $\begin{array}{l}\text { Vacunación } \\
\text { preferencial }\end{array}$ & Observaciones \\
\hline Chile & 14 de marzo del 2020 & Sí & Influenza & $\begin{array}{l}\text { Se priorizará al personal de salud, gestantes, niños de } 6 \\
\text { meses a } 5 \text { años, personas de } 65 \text { años a más y pacientes con } \\
\text { enfermedades crónicas de } 11 \text { a } 64 \text { años. }\end{array}$ \\
\hline Uruguay & 18 de marzo del 2020 & Sí & Influenza & $\begin{array}{l}\text { Se priorizarán las vacunas del esquema primario en los niños de 2, } \\
4,6,12 \text { y } 15 \text { meses de edad, situaciones especiales y vacunación de } \\
\text { gestantes. El proceso de vacunación se dividirá en cuatro etapas: la } \\
\text { primera empezará el } 13 \text { abril y está destinada al personal de salud } \\
\text { y a la población mayor a } 75 \text { años; la segunda, a partir del } 24 \text { abril, a } \\
\text { personas de } 65 \text { años a más; la tercera, a partir del primero de mayo, } \\
\text { a niños de } 6 \text { meses a } 5 \text { años; y la cuarta, a partir del } 8 \text { mayo para el } \\
\text { resto de la población. }\end{array}$ \\
\hline Honduras & 31 de marzo del 2020 & Sí & Influenza & $\begin{array}{l}\text { Suspende las actividades de vacunación masiva por la } \\
\text { pandemia por la COVID-19; sin embargo, en coordinación } \\
\text { con cada región sanitaria se planificará la Jornada Nacional } \\
\text { de Vacunación y Desparasitación } 2020 \text { (11-22 de mayo). Se } \\
\text { priorizará la vacuna contra la influenza para los trabajadores } \\
\text { de la salud, adultos mayores de } 60 \text { años, enfermos crónicos, } \\
\text { gestantes y niños de } 6 \text { a } 23 \text { meses. }\end{array}$ \\
\hline Argentina & 3 de abril del 2020 & Sí & Influenza & $\begin{array}{l}\text { La vacunación se realizará en tres etapas: la primera está } \\
\text { destinada a los adultos mayores de } 65 \text { años y personal de } \\
\text { salud; la segunda, a niños menores de } 2 \text { años y embarazadas; y } \\
\text { la tercera, a personas de } 2 \text { a } 64 \text { años con factores de riesgo. }\end{array}$ \\
\hline Colombia & 3 de abril del 2020 & Sí & Influenza y neumococo & $\begin{array}{l}\text { Si es una persona sospechosa de tener COVID-19, se deriva el } \\
\text { caso al área correspondiente y se difiere la vacunación } 28 \text { días } \\
\text { previa evaluación médica. }\end{array}$ \\
\hline Brasil & 9 de abril del 2020 & $\mathrm{Si}$ & $\begin{array}{l}\text { Influenza, además de } \\
\text { sarampión y fiebre } \\
\text { amarilla para estados } \\
\text { con circulación activa } \\
\text { de la enfermedad }\end{array}$ & $\begin{array}{l}\text { La vacunación se realizará en tres etapas: la primera está } \\
\text { destinada a los adultos mayores de } 60 \text { años y trabajadores de } \\
\text { salud; la segunda, a la población con enfermedades crónicas; } \\
\text { y la tercera, a los niños de } 6 \text { meses a } 6 \text { años, gestantes y } \\
\text { puérperas. Se difiere la vacunación } 14 \text { días si la persona ha } \\
\text { tenido contacto con otras personas con COVID-19. }\end{array}$ \\
\hline Paraguay & 14 de abril del 2020 & Sí & Influenza & $\begin{array}{l}\text { Se realizará en tres etapas: la primera del } 14 \text { al } 27 \text { de abril y } \\
\text { está destinada a personas mayores de } 60 \text { años y personal de } \\
\text { salud; la segunda, del } 28 \text { de abril al } 12 \text { mayo, a niños de } 6 \text { a } 36 \\
\text { meses, niños con enfermedades de base, gestantes y enfermos } \\
\text { crónicos; y la tercera, a los trabajadores de instituciones } \\
\text { públicas o privadas. }\end{array}$ \\
\hline Perú & 20 de abril del 2020 & Sí & $\begin{array}{l}\text { Influenza y } \\
\text { neumococo. Vacuna } \\
\text { contra la hepatitis B, } \\
\text { prioritaria en zonas de } \\
\text { alta prevalencia }\end{array}$ & $\begin{array}{l}\text { Contiene estrategias de vacunación durante el estado de } \\
\text { emergencia y después de este. No se recomienda la vacunación } \\
\text { en pacientes con síntomas de COVID-19; sin embargo, en el } \\
\text { caso de que tenga el diagnóstico se los vacunará dos semanas } \\
\text { después de su alta epidemiológica o clínica. }\end{array}$ \\
\hline Costa Rica & 30 de abril del 2020 & Sí & Influenza & $\begin{array}{l}\text { La inmunización comenzó el } 4 \text { de mayo y terminará el } 3 \text { de julio. } \\
\text { Debido a que no se disponía de la vacuna contra la influenza, el } \\
\text { gobierno envió mensajes de texto para avisarles directamente. } \\
\text { Se vacunará en orden de acuerdo con la primera letra del } \\
\text { primer apellido. Se priorizará la vacunación en personas con } \\
\text { enfermedades crónicas, niños entre } 3 \text { y } 7 \text { años, adultos mayores } \\
\text { de } 59 \text { años a más y todas las personas mayores de } 7 \text { a } 58 \text { años que } \\
\text { forman parte de los grupos de riesgo. }\end{array}$ \\
\hline
\end{tabular}

La OPS recomienda a cada país priorizar la vacunación contra la influenza y el sarampión, y continuar con la vacunación de los recién nacidos, los esquemas primarios y la vacuna contra la influenza y el neumococo en la población de riesgo ${ }^{(1)}$. En los pacientes con la COVID-19, recomienda de manera general no vacunarlos y diferir la administración en los contactos hasta dos semanas después ${ }^{(1)}$. En Latinoamérica los casos de COVID-19 han aumentado, siendo los países más afectados Brasil, Perú y Ecuador ${ }^{(6)}$.

En conclusión, se han programado actividades de inmunización en algunos países latinoamericanos, priorizando la vacuna contra la influenza. Se recomienda que futuros estu- 
dios aborden el impacto de la pandemia por la COVID-19 en las coberturas de vacunación y desarrollen estrategias para futuros escenarios epidemiológicos.

Contribución de los autores: NAA y NMCP participaron en la concepción y el diseño del artículo. NAA y CJPV participaron en la interpretación de datos y revisión crítica del artículo. Todos los autores participaron en la recolección de datos, redacción y aprobación de la versión final.

Financiamiento: Autofinanciado.

Conflictos de interés: CJPV trabaja como médico de enlace científico en Sanofi Pasteur.

\section{REFERENCIAS BIBLIOGRÁFICAS}

1. Organización Panamericana de la Salud, Organización Mundial de la Salud. El programa de inmunización en el contexto de la pandemia de COVID-19 - Marzo 2020 - OPS, OMS [Internet]. 2020 [citado el 7 de mayo de 2020]. Washington, D.C: OPS/OMS; 2020. Disponible en: http://www.paho.org/es/documentos/programa-inmunizacion-contexto-pandemia-covid-19-marzo-2020.

2. Alvarez AMR, Kurtis HJ, Vulanovic L, Hasan H, Ruiz C, Thrush E. The evolution of Vaccination Week in the Americas. Rev Panam Salud Pública. 2018;41:e150. doi: 10.26633/rpsp.2017.150.

3. Nelson R. COVID-19 disrupts vaccine delivery. Lancet Infect Dis. 2020;20(5):546. doi: 10.1016/S1473-3099(20)30304-2.

4. Organización Panamericana de la Salud, Organización Mundial de la Salud. Indicadores básicos 2019: Tendencias de la salud en las Américas [Internet]. Washington, D.C: OPS, OMS; 2019 [citado el 7 de mayo de 2020]. Disponible en: https://iris.paho.org/bitstream/ handle/10665.2/51543/9789275321287_spa.pdf?sequence=7\&isAllowed $=\mathrm{y}$.

5. Organización Panamericana de la Salud, Organización Mundial de la Salud. Actualización Epidemiológica: Sarampión. 17 de abril de 2020 [Internet]. Washington, D.C: OPS, OMS; 2020 [citado el 7 de mayo de 2020]. Disponible en: https://www.paho.org/es/documentos/ actualizacion-epidemiologica-sarampion-17-abril-2020.

6. Johns Hopkins University. Coronavirus COVID-19 Global Cases by the Center for Systems Science and Engineering (CSSE) at Johns Hopkins University (JHU) [Internet]. Johns Hopkins Coronavirus Resource Center. [citado el 7 de mayo de 2020]. Disponible en: https:// coronavirus.jhu.edu/map.html. 